Review Article

\title{
Semilinear Fractional Evolution Inclusion Problem in the Frame of a Generalized Caputo Operator
}

\author{
Adel Lachouri $\mathbb{D}^{1}{ }^{1}$ Abdelouaheb Ardjouni, ${ }^{2}$ Fahd Jarad $\mathbb{D}^{3,4}$ and Mohammed S. Abdo $\mathbb{D}^{5}$ \\ ${ }^{1}$ Laboratory of Applied Mathematics, Department of Mathematics, Annaba University, Annaba, Algeria \\ ${ }^{2}$ Department of Mathematics and Informatics, University of Souk Ahras, Souk Ahras, Algeria \\ ${ }^{3}$ Department of Mathematics, Çankaya University, 06790 Etimesgut, Ankara, Turkey \\ ${ }^{4}$ Department of Medical Research, China Medical University Hospital, China Medical University, Taichung, Taiwan \\ ${ }^{5}$ Department of Mathematics, Hodeidah University, Al-Hodeidah, Yemen \\ Correspondence should be addressed to Fahd Jarad; fahd@cankaya.edu.tr
}

Received 8 August 2021; Revised 9 October 2021; Accepted 11 October 2021; Published 3 November 2021

Academic Editor: Andrea Scapellato

Copyright (C) 2021 Adel Lachouri et al. This is an open access article distributed under the Creative Commons Attribution License, which permits unrestricted use, distribution, and reproduction in any medium, provided the original work is properly cited.

In this paper, we study the existence of solutions to initial value problems for a nonlinear generalized Caputo fractional differential inclusion with Lipschitz set-valued functions. The applied fractional operator is given by the kernel $k(\rho, s)=\xi(\rho)-\xi(s)$ and the derivative operator $\left(1 / \xi^{\prime}(\rho)\right)(d / d \rho)$. The existence result is obtained via fixed point theorems due to Covitz and Nadler. Moreover, we also characterize the topological properties of the set of solutions for such inclusions. The obtained results generalize previous works in the literature, where the classical Caputo fractional derivative is considered. In the end, an example demonstrating the effectiveness of the theoretical results is presented.

\section{Introduction}

Fractional calculus is a field of mathematical analysis that embraces the integrals and derivatives of functions of any real or complex order. For the past few decades, this field has been one of the hand-over-fist sprawling fields of mathematics by virtue of the amazing findings obtained when researchers enrolled the fractional operators in their attempts to construe some problems that arise in nature (see [1-6]). As a matter of fact, the classical fractional calculus consisted of one main integral operator, namely, the Riemann-Liouville fractional integral, and two fractional derivatives, namely, the Riemann-Liouville and Caputo derivatives. Because of the penurious number of operators, researchers were compelled to discover and develop new fractional operators that allow them to better comprehend the world around them. In the last 10 years or so, new fractional operators have been introduced and wielded on many occasions. One can touch upon the operators in [7-13]. It should be noted that some of these operators are an extension or generalization of the RiemannLiouville integrals and Riemann-Liouville and Caputo deriv- atives that are nonlocal but singular kerneled. The others are brand-new ones, and they are nonlocal and contain nonsingular kernels.

One of the main applications of advanced fractional calculus is the theory of fractional evolution inclusions since they are abstract formulations for numerous problems arising in physics and engineering. Evolution equations and inclusions are commonly applied to describe the systems and models that change or evolve over time. Many studies have investigated the existence and uniqueness of solutions for fractional evolution problems based on semigroup and fixed point theories; e.g., Bedi et al. [14] studied the existence of mild solutions for Hilfer-type fractional neutral impulsive evolution problems in a Banach space. The same author, with others in [15], discussed the stability and controllability results for Hilfer-type fractional evolution equations in a Banach space. The existence of saturated mild (and global) solutions of Caputo-type fractional semilinear evolution problems with noncompact semigroups has been obtained in Banach spaces by Chen et al. [16]. The authors in [17] studied the existence of local (and global) solutions and the 
uniqueness of a mild solution to fractional semilinear evolution problems with compact (and noncompact) semigroups in Banach spaces. Zhou and Jiao [18] considered a class of nonlocal Cauchy problems for a Caputo-type fractional neutral evolution problem to investigate the existence and uniqueness of mild solutions. For differential equationsinclusions governed by Cauchy conditions or boundary conditions, the issue of importance is to tackle the existence, uniqueness, and stability of their solutions. These properties constitute the most essential parts of the analysis of these equations. There are heaps of papers that went about the existence, uniqueness, and stability results of differential equations-inclusions within the scope of a variety of fractional derivatives based on different types of fixed point techniques. For instance, Abdo et al. [19] have proven the existence and different types of stability of solutions for $\psi$ Hilfer-type fractional integro-problems. The authors in [20] investigated some existence results of Caputo-type fractional neutral inclusions by using weak topology. The existence of solutions for a generalized Caputo-type fractional differential inclusion problem with integral boundary conditions has been studied by Belmor et al. [21]. In [22], the authors discussed the existence of solutions for a Caputotype fractional for higher-order fractional inclusion problems. Lachouri et al. [23] considered a nonlinear Hilfer-type nonlocal fractional inclusion problem to prove the existence results, taking into account the convex and nonconvex values of the nonlinear term.

Recently, Chen et al. [16] proved the existence of saturated mild solutions and global mild solutions for fractional evolution equations of the type

$$
\left\{\begin{array}{l}
{ }^{C} D^{r_{1}} \sigma(\rho)+\mathscr{A} \sigma(\rho)=h(\rho, \sigma(\rho)), \rho \geq 0, \\
\sigma(0)=\sigma_{0} \in \mathscr{X},
\end{array}\right.
$$

$\in(0,1), \mathscr{A}: \mathfrak{D}(\mathscr{A}) \subset \mathscr{X} \longrightarrow \mathscr{X}$ is a closed linear operator and $-\mathscr{A}$ generates a uniformly bounded $C_{0}$-semigroup $\{\mathscr{T}(\rho)\}_{\rho \geq 0}$ in $\mathscr{X}$, and $h:[0, \infty) \times \mathscr{X} \longrightarrow \mathscr{X}$ is the given function.

In [17], Suechoei and Ngiamsunthorn studied the following fractional evolution equations:

$$
\left\{\begin{array}{l}
{ }^{C} D_{a+}^{r_{1} ; \xi} \sigma(\rho)=\mathscr{A} \sigma(\rho)+h(\rho, \sigma(\rho)), \quad \rho \in(0, T], \\
\sigma(0)=\sigma_{0}, \quad \sigma_{0} \in X,
\end{array}\right.
$$

where ${ }^{C} D_{\mathfrak{a}++}^{r_{1} ; \xi}$ is the $\xi$-Caputo FD of order $r_{1} \in(0,1), \mathscr{A}$ is the infinitesimal generator of a $C_{0}$-semigroup of uniformly bounded linear operators $\{\mathscr{T}(\rho)\}_{\rho \geq 0}$ on $\mathscr{X}$, and $h:[0, \infty)$ $\times \mathscr{X} \longrightarrow \mathscr{X}$ is the given function.

Motivated by the aforementioned works and inspired by [17], we consider the following fractional evolution inclusion involving $\xi$-Caputo FD:

$$
\left\{\begin{array}{l}
{ }^{C} D_{a+}^{r_{1} ; \xi} \sigma(\rho) \in \mathscr{A} \sigma(\rho)+f(\rho, \sigma(\rho)), \quad \rho \in(0, T], \\
\sigma(0)=\sigma_{0}, \quad \sigma_{0} \in X,
\end{array}\right.
$$

where $f:[0, \mathfrak{I}] \times \mathscr{X} \longrightarrow \mathcal{O}(\mathscr{X})$ is a set-valued map (svm) from $[0, \mathfrak{I}] \times \mathscr{X}$ to the family of $\mathcal{O}(\mathscr{X}) \subset \mathscr{X}$ and $\mathscr{X}$ is a Banach space with the norm $\|$.$\| .$

The novelty of the present work is choosing a more general operator than the classical fractional operators. More precisely, problem (3) is reduced to a Caputo-type problem, for $\xi(\rho)=\rho$; Hadamard-Caputo-type problem, for $\xi(\rho)=$ $\log (\rho)$; and Katugampola-Caputo-type problem, for $\xi(\rho)$ $=\rho^{\delta}, \delta>0$. In addition, we describe the topological properties of the considered solution in the present work.

In this paper, we aim to prove the existence of mild solutions for problem (3) involving the generalized Caputo derivative using the fixed point theorem (FPT) of Nadler and Covitz and to characterize the topological properties of the set of solutions for such inclusions.

The acquired results are more general and cover many of the parallel problems that contain special cases of function $\xi$, such as $[16,18]$.

\section{Preliminary Notions}

2.1. Fractional Calculus (FC). In this portion, we introduce several basic notions of FC and necessary lemmas that will be needed in such a study.

Let $\mathfrak{I}^{*}=[0, \mathfrak{I}]$. Denoted by $\mathscr{E}=C\left(\mathfrak{J}^{*}, \mathscr{X}\right)$, we have the Banach space of continuous functions $\sigma: \mathfrak{I}^{*} \longrightarrow \mathscr{X}$ with

$$
\|\sigma\|_{\mathscr{E}}=\sup \left\{\|\sigma(\rho)\|: \rho \in \mathfrak{J}^{*}\right\} .
$$

Let $\sigma, \xi \in C^{n}\left(\mathfrak{I}^{*}, \mathbb{R}\right)$ such that $\xi$ is increasing and $\xi^{\prime}(\rho)$ $\neq 0, \forall \rho \in \mathfrak{I}^{*}$, and

$$
\mathscr{M}=\sup _{\rho \in[0, \infty)}\|\mathscr{T}(\rho)\|<\infty .
$$

Definition 1 (see [24]). The $\xi$-Riemann-Liouville FI of a function $\sigma$ of order $r_{1}$ is defined by

$$
I_{\mathfrak{a}+}^{r_{1} ; \xi} \sigma(\rho)=\frac{1}{\Gamma\left(r_{1}\right)} \int_{\mathfrak{a}}^{\rho} \xi^{\prime}(v)(\xi(\rho)-\xi(v))^{r_{1}-1} \sigma(v) d v .
$$

Definition 2 (see [24]). The $\xi$-Riemann-Liouville FD of order $r_{1}$ for a function $\sigma$ is given by

$$
D_{\mathfrak{a}+}^{r_{1} ; \xi} \sigma(\rho)=\left(\frac{1}{\xi^{\prime}(\rho)} \frac{d}{d \rho}\right)^{n} I_{\mathfrak{a}+}^{\left(n-r_{1}\right) ; \xi} \sigma(\rho),
$$

where $n=\left[r_{1}\right]+1, n \in \mathbb{N}$.

Definition 3 (see [13]). The $\xi$-Caputo FD of a function $\sigma \epsilon$ $C^{n}\left(\mathfrak{J}^{*}, \mathbb{R}\right)$ of order $r_{1}$ is described by

$$
{ }^{C} D_{\mathfrak{a}_{+}}^{r_{1} ; \xi} \sigma(\rho)=I_{\mathfrak{a}+}^{\left(n-r_{1}\right) ; \xi} \sigma^{[n]}(\rho),
$$


where $\sigma^{[n]}(\rho)=\left(\left(1 / \xi^{\prime}(\rho)\right)(d / d \rho)\right)^{n} \sigma(\rho)$ and $n=\left[r_{1}\right]+1$, $n \in \mathbb{N}$.

Lemma 4 (see $[11,25])$. Let $r_{1}, r_{2}, \mu>0$. Then,

$$
\begin{aligned}
& I_{\mathfrak{a}+}^{r_{1} ; \xi}(\xi(\rho)-\xi(\mathfrak{a}))^{r_{2}-1}(\rho)=\frac{\Gamma\left(r_{2}\right)}{\Gamma\left(r_{1}+r_{2}\right)}(\xi(\rho)-\xi(\mathfrak{a}))^{r_{1}+r_{2}-1}, \\
& { }^{C} D_{\mathfrak{a}+}^{r_{1} ; \xi}(\xi(\rho)-\xi(\mathfrak{a}))^{r_{2}-1}(\rho)=\frac{\Gamma\left(r_{2}\right)}{\Gamma\left(r_{2}-r_{1}\right)}(\xi(\rho)-\xi(\mathfrak{a}))^{r_{1}+r_{2}-1} .
\end{aligned}
$$

Lemma 5 (see [13]). If $\sigma \in C^{n}\left(\mathfrak{J}^{*}, \mathbb{R}\right), r_{1} \in(n-1, n)$, and $r_{2} \in(0,1)$, then

$$
I_{\mathfrak{a}+}^{r_{1} ; \xi C} D_{\mathfrak{a}+}^{r_{1} ; \xi} \sigma(\rho)=\sigma(\rho)-\sum_{k=0}^{n-1} \frac{\sigma^{[n]}\left(\mathfrak{a}^{+}\right)}{k !}(\xi(\rho)-\xi(\mathfrak{a}))^{k}
$$

In particular, given $r_{1} \in(0,1)$, we have

$$
I_{\mathfrak{a}+}^{r_{1} ; \xi C} D_{\mathfrak{a}+}^{r_{1} ; \xi} \sigma(\rho)=\sigma(\rho)-\sigma(\mathfrak{a}) .
$$

Definition 6 (see [11]). Let $\sigma, \xi:[\mathfrak{a}, \infty) \longrightarrow \mathbb{R}$ be real-valued functions. The generalized Laplace transform of $\sigma$ is defined by

$$
\mathfrak{L}\{\sigma(\rho)\}(v)=\int_{\mathfrak{a}}^{\rho} e^{-v(\xi(\rho)-\xi(\mathfrak{a}))} \sigma(\rho) \xi^{\prime}(\rho) d \rho, \quad \text { for all } v .
$$

Theorem 7 (see [11]). Let $r_{1}>0$ and $\varkappa$ be a piecewise continuous function on each interval $[\mathfrak{a}, \rho]$ and $\xi(\rho)$-exponential order. Then,

$$
\mathbb{L}\left\{I_{\mathfrak{a}+}^{r_{1} ; \xi} \sigma(\rho)\right\}(v)=\frac{I_{\mathfrak{a}+}^{r_{1} ; \xi} \sigma(\rho)}{v^{r_{1}}} .
$$

Definition 8 (see $[26,27])$. Let $r_{1} \in(0,1)$ and $\rho \in \mathbb{C}$. The Wright-type function is defined by

$$
\begin{aligned}
\vartheta_{r_{1}}(\rho) & =\sum_{\mathfrak{m}=0}^{\infty} \frac{(-\rho)^{\mathfrak{m}}}{\mathfrak{m} ! \Gamma\left(-r_{1} \mathfrak{m}+1-r_{1}\right)} \\
& =\sum_{\mathfrak{m}=0}^{\infty} \frac{(-\rho)^{\mathfrak{m}} \Gamma\left(r_{1}(\mathfrak{m}+1)\right) \sin \left(\pi r_{1}(\mathfrak{m}+1)\right)}{\mathfrak{m} !}
\end{aligned}
$$

Proposition 9 (see [26, 27]). The Wright function $\vartheta_{r_{1}}$ is an entire function and has the properties listed below:

(1) $\vartheta_{r_{1}} \geq 0$ for $\rho \geq 0$ and $\int_{0}^{\infty} \vartheta_{r_{1}}(\rho) d \rho=1$

(2) $\int_{0}^{\infty} \vartheta_{r_{1}}(\rho) \rho^{\alpha} d \rho=\Gamma(1+\alpha) / \Gamma\left(1+\alpha r_{1}\right)$, for $\alpha>-1$

(3) $\int_{0}^{\infty} \vartheta_{r_{1}}(\rho) e^{-k \rho} d \rho=E_{r_{1}}(-k), k \in \mathbb{C}$

(4) $\alpha \int_{0}^{\infty} \rho \vartheta_{r_{1}}(\rho) e^{-k \rho} d \rho=E_{r_{1}, r_{1}}(-k), k \in \mathbb{C}$
Next, we recall some conceptions concerning the semigroups of linear operators. For more details, see [28, 29].

For strongly continuous semigroups (i.e., $\mathrm{C}_{0}$-semigroup $\{\mathscr{T}(\rho)\}_{\rho \geq 0}$ on $\left.\mathscr{X}\right)$, we define the generator

$$
\mathscr{A} \sigma=\lim _{\rho \longrightarrow 0^{+}} \frac{\mathscr{T}(\rho) \sigma-\sigma}{\sigma}, \quad \sigma \in \mathscr{X} .
$$

By $\mathfrak{D}(\mathscr{A})$, we denote the domain of $\mathscr{A}$, that is,

$$
\mathfrak{D}(\mathscr{A})=\left\{\sigma \in \mathscr{X}: \lim _{\rho \longrightarrow 0^{+}} \frac{\mathscr{T}(\rho) \sigma-\sigma}{\sigma} \text { exists }\right\} .
$$

Lemma 10 (see $[28,29])$. Let $\{\mathscr{T}(\rho)\}_{\rho \geq 0}$ be a $C_{0}$-semigroup; then, there exist constants $\mathfrak{c}>0$ and $\mathfrak{a} \geq 0$ such that

$$
\|\mathscr{T}(\rho)\| \leq \mathfrak{C} e^{\mathfrak{a} \tau}, \quad \forall \rho \geq 0 .
$$

Lemma 11 (see $[28,29])$. A linear operator $\mathscr{A}$ is the infinitesimal generator of a $\mathrm{C}_{0}$-semigroup iff

(1) $\mathscr{A}$ is closed, and $\mathfrak{D}(\mathscr{A})=\mathscr{X}$

(2) The resolvent set $\mathscr{R}(\mathscr{A})$ of $\mathscr{A}$ contains $\mathbb{R}^{+}$, and for every $\lambda \in \mathbb{R}^{+}$, we have

$$
\|R(\lambda, \mathscr{A})\| \leq \frac{1}{\lambda}
$$

where $R(\lambda, \mathscr{A})=\left(\lambda^{r_{1}} I-\mathscr{A}\right)^{-1} \sigma=\int_{0}^{\infty} e^{-\lambda \tau} \mathscr{T}(\rho) \sigma d \rho$.

In relation to problem (3), we need the following lemma which was proven in [17].

Lemma 12. The mild solution of IVPs

$$
\left\{\begin{array}{l}
{ }^{C} D_{a+}^{r_{1} ; \xi} \sigma(\rho)=\mathscr{A} \sigma(\rho)+q(\rho, \sigma(\rho)), \quad \rho \in(0, T], \\
\sigma(0)=\sigma_{0},
\end{array}\right.
$$

is obtained as

$$
\sigma(\rho)=\mathcal{S}^{r_{1} ; \xi}(\rho, 0) \sigma_{0}+\int_{0}^{\rho} \xi^{\prime}(v)(\xi(\rho)-\xi(v))^{r_{1}-1} \mathscr{T}^{r_{1} ; \xi}(\rho, v) q(v, \sigma(v)) d v,
$$

where

$$
\begin{aligned}
\mathcal{S}^{r_{1} ; \xi}(\rho, v) \sigma & =\int_{0}^{\infty} \vartheta_{r_{1}}(\theta) \mathscr{T}\left((\xi(\rho)-\xi(v))^{r_{1}} \theta\right) \sigma d \theta, \\
\mathscr{T}^{r_{1} ; \xi}(\rho, v) \sigma & =r_{1} \int_{0}^{\infty} \theta \vartheta_{r_{1}}(\theta) \mathscr{T}\left((\xi(\rho)-\xi(v))^{r_{1}} \theta\right) \sigma d \theta,
\end{aligned}
$$

for $0 \leq v \leq \rho \leq \mathfrak{I}$. 
Lemma 13 (see [18]). The operators $\mathscr{T}^{r_{1} ; \xi}$ and $\mathcal{S}^{r_{1} ;}$ have the following properties:

(1) For any fixed $\rho \geq v \geq 0, \mathscr{T}^{r_{1} ; \xi}$ and $\mathcal{S}^{r_{1} ; \xi}$ are bounded linear operators with

$$
\begin{gathered}
\left\|\mathcal{S}^{r_{1} ; \xi}(\rho, v)(\sigma)\right\| \leq \mathscr{M}\|\sigma\|, \\
\left\|\mathscr{T}^{r_{1} ; \xi}(\rho, v)(\sigma)\right\| \leq \frac{r_{1} \mathscr{M}}{\Gamma\left(1+r_{1}\right)}=\frac{\mathscr{M}}{\Gamma\left(r_{1}\right)}\|\sigma\|, \quad \forall \sigma \in \mathscr{X} .
\end{gathered}
$$

(2) $\mathscr{T}^{r_{1} ; \xi}$ and $\mathcal{S}^{r_{1} ; \xi}$ are strongly continuous $\forall \rho \geq v \geq 0$; that is, for every $\omega \in \mathscr{X}$ and $0 \leq v \leq \rho_{1} \leq \rho_{2} \leq \mathfrak{I}$, we have

$$
\begin{aligned}
& \left\|\mathcal{S}^{r_{1} ; \xi}\left(\rho_{2}, v\right) \sigma-\mathcal{S}^{r_{1} ;}\left(\rho_{1}, v\right) \sigma\right\| \longrightarrow 0, \\
& \left\|\mathscr{T}^{r_{1} ; \xi}\left(\rho_{2}, v\right) \sigma-\mathscr{T}^{r_{1} ; \xi}\left(\rho_{1}, v\right) \sigma\right\| \longrightarrow 0,
\end{aligned}
$$

as $\rho_{1} \longrightarrow \rho_{2}$.

(3) If $\mathscr{T}(\rho)$ is a compact operator $\forall \rho>0$, then $\mathscr{T}^{r_{1} ; \xi}(\rho, v)$ and $\mathcal{S}^{r_{1} ; \xi}(\rho, v)$ are compact for all $\rho, v>0$.

(4) The operators $\mathscr{T}^{r_{1} ; \xi}(\rho, v)$ and $\mathcal{S}^{r_{1} ; \xi}(\rho, v)$ are continuous in the uniform operator topology.

\section{Main Results}

In what follows, we will utilize the notation $\mathcal{O}_{c p, c}(\mathscr{X})=\{\mathcal{N}$ $\in \mathcal{O}(v): \mathscr{N}$ is compact and convex $\}$ for a normed space $\mathscr{X}$. For more information about the svm, we refer the reader to $[30,31]$.

Definition 14. A function $\sigma \in C^{1}\left(\mathfrak{\Im}^{*}, \mathscr{X}\right)$ is a solution of (3), if $\exists \wp^{\sim} \in L^{1}\left(\mathfrak{I}^{*}, \mathscr{X}\right)$ with $\wp^{\sim}(\rho) \in f(\rho, \sigma) \forall \rho \in \mathfrak{I}^{*}$ fulfilling the initial condition

$$
\begin{gathered}
\sigma(0)=\sigma_{0}, \\
\sigma(\rho)=\mathcal{S}^{r_{1} ; \xi}(\rho, 0) \sigma_{0}+\int_{0}^{\rho} \xi^{\prime}(v)(\xi(\rho)-\xi(v))^{r_{1}-1} \mathscr{T}^{r_{1} ; \xi}(\rho, v) \wp^{\sim}(v) d v .
\end{gathered}
$$

The first outcome treats the nonconvex $f$ based on the theorem of Covitz and Nadler [32].

Let $(\sigma, d)$ be a metric space induced from the normed space $(\sigma,\|\|$.$) . Consider \mathscr{H}_{d}: \mathcal{O}(\sigma) \times \mathcal{O}(\sigma) \longrightarrow \mathbb{R}^{+} \cup\{\infty\}$ defined by

$$
\mathscr{H}_{d}(\tilde{C}, \tilde{D})=\max \left\{\sup _{\tilde{c} \in \tilde{C}} d(\tilde{\mathcal{C}}, \tilde{D}), \sup _{\tilde{d} \in \tilde{D}} d(\tilde{C}, \tilde{d})\right\},
$$

where $d(\tilde{C}, \tilde{d})=\inf _{\tilde{c} \in \tilde{C}} d(\tilde{c}, \tilde{d})$ and $d(\tilde{c}, \tilde{D})=\inf _{\tilde{d} \in \tilde{D}} d(\tilde{c}, \tilde{d})$. Then, $\left(\mathscr{O}_{b, c l}(\sigma), \mathscr{H}_{d}\right)$ is a metric space (see [33]).

Definition 15. A svm $\mathfrak{F}: \mathscr{X} \longrightarrow \mathcal{O}_{c l}(\mathscr{X})$ is $v$-Lipschitz iff $\exists v>0$ such that

$$
\mathscr{H}_{d}(\mathfrak{F}(\sigma), \mathfrak{F}(\rho)) \leq v d(\sigma, \rho), \quad \forall \sigma, \rho \in \mathscr{X} .
$$

Particularly, if $v<1$, then $\mathfrak{F}$ is a contraction.

Theorem 16. Let

$$
\rho=\frac{\mathscr{M}}{\Gamma\left(r_{1}+1\right)}(\xi(\mathfrak{I})-\xi(0))^{r_{1}},
$$

and assume that

(As1) $f: \mathfrak{I}^{*} \times \mathscr{X} \longrightarrow \mathcal{O}_{c p}(\mathscr{X})$ such that $f(., \sigma): \mathfrak{J}^{*} \longrightarrow$ $\mathcal{O}_{c p}(\mathscr{X})$ is measurable for any $\sigma \in \mathscr{X}$.

(As2) $\mathscr{H}_{d}(f(\rho, \sigma), f(\rho, \bar{\sigma})) \leq \mathfrak{z}(\rho)\|\sigma-\bar{\sigma}\|$ for (a.e.) all $\rho$ $\in \mathfrak{J}^{*}$ and $\sigma, \bar{\sigma} \in \mathbb{R}$ with $\mathfrak{z} \in C\left(\mathfrak{J}^{*}, \mathbb{R}^{+}\right)$and $d(0, f(\rho, 0)) \leq \mathfrak{z}$ ( $\rho$ ) for (a.e.) all $\rho \in \mathfrak{J}^{*}$.

Then, (3) possesses at least one solution on $\mathfrak{\Im}^{*}$ if

$$
\rho\|\mathfrak{z}\|_{\infty}<1
$$

Proof. At first, to switch problem (3) into a FP, we formulate $\mathfrak{F}: \mathscr{E} \longrightarrow \mathscr{O}(\mathscr{E})$ as

$$
\begin{aligned}
\mathfrak{F}(\sigma) & =\{\phi \in \mathscr{E}: \phi(\rho) \\
& =\left\{\mathcal{S}^{r_{1} ; \xi}(\rho, 0) \sigma_{0}+\int_{0}^{\rho} \xi^{\prime}(v)(\xi(\rho)-\xi(v))^{r_{1}-1} \mathscr{T}^{r_{1} ; \xi}(\rho, v) \tilde{\mathscr{f}}(v) d v\right\},
\end{aligned}
$$

for $\mathfrak{\wp}^{\sim} \in \mathscr{R}_{f, \sigma}$. The solution of (3) is obviously an FP of $\mathfrak{F}$. The following are the steps in the proofing process.

By virtue of assumption (As1) and [34] (Theorem III.6), $f$ has a measurable selection, and thus, $\mathscr{R}_{f, \sigma} \neq \varnothing$. In the sequel, we demonstrate that $\mathfrak{F}: \mathscr{E} \longrightarrow \mathcal{O}(\mathscr{E})$ defined in (29) fulfills the assumptions of FPT of Covitz and Nadler. First, we show that $\mathfrak{F}(\sigma)$ is closed for every $\sigma \in \mathscr{E}$. Let $\left\{u_{n}\right\}_{n=0}^{\infty} \in \mathfrak{F}(\sigma)$ such that $\mathfrak{u}_{n} \longrightarrow \mathfrak{t}(n \longrightarrow \infty)$ in $\mathscr{E}$. Then,

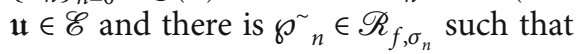

$$
\mathfrak{u}_{n}(\rho)=\mathcal{S}^{r_{1} ; \xi}(\rho, 0) \sigma_{0}+\int_{0}^{\rho} \xi^{\prime}(v)(\xi(\rho)-\xi(v))^{r_{1}-1} \mathscr{T}^{r_{1} ; \xi}(\rho, v) \mathscr{\mho}_{n}{ }_{n}(v) d v, \quad \rho \in \mathfrak{I}^{*} .
$$

Accordingly, there is a subsequence $\tilde{\wp}_{n}$ that converges to $\tilde{\wp}$ in $L^{1}\left(\mathfrak{I}^{*}, \mathscr{X}\right)$ because $f$ has compact values. As an outcome, $\wp^{\sim} \in \mathscr{R}_{f, \sigma}$, and we get 


$$
\begin{aligned}
& \mathfrak{u}_{n}(\rho) \longrightarrow \mathfrak{u}(\rho)=\mathcal{S}^{r_{1} ; \xi}(\rho, 0) v_{0} \\
& \quad+\int_{0}^{\rho} \xi^{\prime}(v)(\xi(\rho)-\xi(v))^{r_{1}-1} \mathscr{T}^{r_{1} ; \xi}(\rho, v) \wp^{\sim}(v) d v, \quad \forall \rho \in \mathfrak{J}^{*} .
\end{aligned}
$$

Hence, $u \in \mathfrak{F}(\sigma)$.

It remains to demonstrate that there is a $\theta \in(0,1)$ $\left(\theta=\rho\|\mathfrak{z}\|_{\infty}\right)$ such that

$$
H_{d}(\mathfrak{F}(\sigma), \mathfrak{F}(\bar{\sigma})) \leq \theta\|\sigma-\bar{\sigma}\|, \quad \forall \sigma, \bar{\sigma} \in \mathscr{E} .
$$

Let $\sigma, \bar{\sigma} \in \mathscr{E}$ and $\phi_{1} \in \mathfrak{F}(\sigma)$. Then, there exists $\wp^{\sim}{ }_{1}(\rho) \in f(\rho, \sigma(\rho))$ such that

$\phi_{1}(\rho)=\mathcal{S}^{r_{1} ; \xi}(\rho, 0) \sigma_{0}+\int_{0}^{\rho} \xi^{\prime}(v)(\xi(\rho)-\xi(v))^{r_{1}-1} \mathscr{T}^{r_{1} ; \xi}(\rho, v) \tilde{\gamma}_{1}{ }_{1}(v) d v$.

By (As2), we have

$$
\mathscr{H}_{d}(f(\rho, \sigma), f(\rho, \bar{\sigma})) \leq \mathfrak{z}(\rho)\|\sigma(\rho)-\bar{\sigma}(\rho)\| .
$$

So, there exists $\widehat{\varsigma}(\rho) \in f(\rho, \bar{\sigma})$ such that

$$
\left\|\gamma^{\sim}{ }_{1}(\rho)-\widehat{\varsigma}\right\| \leq \mathfrak{z}(\rho)\|\sigma(\rho)-\bar{\sigma}(\rho)\|, \quad \rho \in \mathfrak{I}^{*} .
$$

As follows, we build a svm $\chi: \mathfrak{I}^{*} \longrightarrow \mathscr{O}(\mathscr{X})$, where

$$
\chi(\rho)=\left\{\widehat{\varsigma} \in \mathscr{X}:\left\|\wp^{\sim}{ }_{1}(\rho)-\widehat{\varsigma}\right\| \leq \mathfrak{z}(\rho)\|\sigma(\rho)-\bar{\sigma}(\rho)\|\right\} .
$$

From [34] (Proposition III.4), the svm $\chi(\rho) \cap f(\rho, \bar{\sigma})$ is measurable. We now select the function $\wp_{\gamma_{2}}{ }_{2}(\rho) \in f(\rho, \bar{\sigma})$ such that

$$
\left\|\widetilde{\gamma}_{1}(\rho)-\wp^{\sim}{ }_{2}(\rho)\right\| \leq \mathfrak{z}(\rho)\|\sigma(\rho)-\bar{\sigma}(\rho)\|, \quad \forall \rho \in \mathfrak{I}^{*} .
$$

We define

$\phi_{2}(\rho)=\mathcal{\delta}^{r_{1} ; \xi}(\rho, 0) \sigma_{0}+\int_{0}^{\rho} \xi^{\prime}(v)(\xi(\rho)-\xi(v))^{r_{1}-1} \mathscr{T}^{r_{1} ; \xi}(\rho, v) \mathscr{F}_{2}{ }_{2}(v) d v, \quad \forall \rho \in \mathfrak{I}^{*}$.

As a consequence, we get

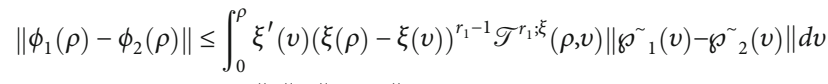

$$
\begin{aligned}
& \leq \frac{\mathscr{M}\left\|_{\mathfrak{z}}\right\|_{\infty}\|\sigma-\bar{\sigma}\|_{\mathscr{\mathscr { C }}}}{\Gamma(\xi(\mathfrak{T})-\xi(0))^{r_{1}}} .
\end{aligned}
$$

Therefore,

$$
\left\|\phi_{1}-\phi_{2}\right\|_{\mathscr{E}} \leq \rho\|\mathfrak{z}\|_{\infty}\|\sigma-\bar{\sigma}\|_{\mathscr{E}} .
$$

By the analogous relation, obtained by interchanging the roles of $\sigma$ and $\bar{\sigma}$, we get

$$
\mathscr{H}_{d}(\mathfrak{F}(\sigma), \mathfrak{F}(\bar{\sigma})) \leq \rho\left\|_{\mathfrak{z}}\right\|_{\infty}\|\sigma-\bar{\sigma}\|_{\mathscr{E}} .
$$

Because $\mathfrak{F}$ is a contraction, we conclude that it has a FP, which is a solution of (3) based on the Covitz and Nadler theorem.

Next, we study the topological properties of the set of solutions of problem (3).

\section{Theorem 17. Assume that}

(As3) $\mathscr{T}(\rho)$ is a compact operator for each $\rho>0$.

(As4) $f: \mathfrak{J}^{*} \times \mathscr{X} \longrightarrow \mathcal{O}_{c p, c}(\mathscr{X})$ is a $L^{1}$-Carathéodory setvalued map.

(As5) $\exists \tilde{\ell}_{1} \in C\left(\mathfrak{\Im}^{*}, \mathbb{R}^{+}\right)$and a nondecreasing $\tilde{\ell}_{2} \in C\left(\mathbb{R}^{+}\right.$, $\mathbb{R}^{+}$) such that

$\|f(\rho, \sigma)\|_{\mathscr{O}}=\sup \{\|\gamma\|: \gamma \in f(\rho, \sigma)\} \leq \tilde{\ell}_{1}(\rho) \tilde{\ell}_{2}\left(\|\sigma\|_{\mathscr{E}}\right), \quad \forall(\rho, \sigma) \in \mathfrak{I}^{*} \times \mathscr{X}$.

Then, the set of solutions of (3) is convex and relatively compact.

Proof. We consider the operator $\mathfrak{F}$ defined in (29) and demonstrate in the following steps that the set of solutions of (3) is convex and relatively compact in $\mathscr{E}$.

(Step 1) The svm $\mathfrak{F}(\sigma)$ is convex for every $\sigma \in \mathscr{E}$.

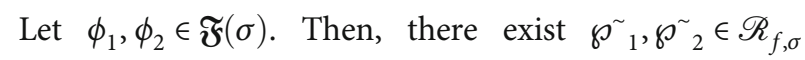
such that

$$
\begin{aligned}
\phi_{i}(\rho)= & \delta^{r_{1} ; \xi}(\rho, 0) \sigma_{0}+\int_{0}^{\rho} \xi^{\prime}(v)(\xi(\rho) \\
& -\xi(v))^{r_{1}-1} \mathscr{T}^{r_{1} ; \xi}(\rho, v) \boldsymbol{\gamma}_{i}{ }_{i}(v) d v, \quad i=1,2, \forall \rho \in \mathfrak{J}^{*} .
\end{aligned}
$$

Let $\varkappa \in[0,1]$. Then, for every $\rho \in \mathfrak{I}^{*}$,

$$
\begin{aligned}
{\left[\varkappa \phi_{1}\right.} & \left.+(1-\varkappa) \phi_{2}\right](\rho)=\mathcal{S}^{r_{1} ; \xi}(\rho, 0) \sigma_{0} \\
& +\int_{0}^{\rho} \xi^{\prime}(v)(\xi(\rho)-\xi(v))^{r_{1}-1} \mathscr{T}^{r_{1} ; \xi}(\rho, v)\left[\varkappa \widetilde{\sigma}_{1}^{\sim_{1}}(v)+(1-\varkappa) \mathscr{\mho}_{2}{ }_{2}(v)\right] d v
\end{aligned}
$$

Since $f$ has convex values, $\mathscr{R}_{f, \sigma}$ is convex and $[\varkappa$ $\left.\boldsymbol{\wp}^{\sim}{ }_{1}(\rho)+(1-\varkappa) \boldsymbol{\wp}_{2}{ }_{2}(\rho)\right] \in \mathscr{R}_{\mathfrak{f}, \sigma}$. Thus, $\varkappa \phi_{1}+(1-\varkappa) \phi_{2} \in \mathfrak{F}(\sigma)$.

(Step 2) $\mathfrak{F}\left(B_{r}\right)$ is bounded on bounded sets of $\mathcal{S}$.

For a constant $r \in \mathbb{R}^{+}$, let $B_{r}=\left\{\sigma \in \mathcal{S}:\|\sigma\|_{\mathscr{C}} \leq r\right\}$ be a bounded set in $\mathcal{S}$. Then, for each $\phi \in \mathfrak{F}(\sigma)$ and $v \in B_{r}$, there exists $\wp^{\sim} \in \mathscr{R}_{f, \sigma}$ such that

$\phi(\rho)=\mathcal{S}^{r_{1} ; \xi}(\rho, 0) \sigma_{0}+\int_{0}^{\rho} \xi^{\prime}(v)(\xi(\rho)-\xi(v))^{r_{1}-1} \mathscr{T}^{r_{1} ; \xi}(\rho, v) \boldsymbol{\gamma}^{\sim}(v) d v$. 
Under hypothesis (As4) and Lemma 13 (1), for any $\rho$ $\in \mathfrak{I}^{*}$, we attain

$$
\begin{aligned}
\|\phi(\rho)\| \leq & \left\|\mathcal{S}^{\mathcal{S}_{1} ; \xi}(\rho, 0)\right\|\left\|\sigma_{0}\right\| \\
& +\int_{0}^{\rho} \xi^{\prime}(v)(\xi(\rho)-\xi(v))^{r_{1}-1}\left\|\mathcal{T}^{r_{1} ; \xi}(\rho, v)\right\|\left\|\boldsymbol{\gamma}^{\sim}(v)\right\| d v \\
\leq & \mathscr{M}\left\|\sigma_{0}\right\|+\frac{\left\|\tilde{\ell}_{1}\right\|_{\infty} \tilde{\ell}_{2}(r) \mathscr{M}}{\Gamma\left(r_{1}+1\right)}(\xi(\mathfrak{I})-\xi(0))^{r_{1}} .
\end{aligned}
$$

Thus,

$$
\|\phi\|_{\mathscr{E}} \leq \mathscr{M}\left\|\sigma_{0}\right\|+\rho\left\|\tilde{\ell}_{1}\right\|_{\infty} \tilde{\ell}_{2}(r)
$$

where $\rho$ is defined in (27).

(Step 3) $\mathfrak{F}$ sends bounded sets of $\mathscr{E}$ into equicontinuous sets.

Let $\sigma \in B_{r}$ and $\phi \in \mathfrak{F}(\sigma)$. Then, there is a function $\wp^{\sim} \in \mathscr{R}_{f, \sigma}$ such that

$$
\phi(\rho)=\mathcal{S}^{r_{1} ; \xi}(\rho, 0) \sigma_{0}+\int_{0}^{\rho} \xi^{\prime}(v)(\xi(\rho)-\xi(v))^{r_{1}-1} \mathscr{T}^{r_{1} ; \xi}(\rho, v) \wp^{\sim}(v) d v .
$$

Let $\rho_{1}, \rho_{2} \in \mathfrak{I}^{*}, \rho_{1}<\rho_{2}$. Then,

$$
\begin{aligned}
& \left\|\phi\left(\rho_{2}\right)-\phi\left(\rho_{1}\right)\right\| \leq\left\|\delta^{r_{1} ; \xi}\left(\rho_{2}, 0\right) \sigma_{0}-\delta^{r_{1} ; \xi}\left(\rho_{1}, 0\right) \sigma_{0}\right\| \\
& +\| \int_{0}^{\rho_{1}} \xi^{\prime}(v)\left(\xi\left(\rho_{1}\right)-\xi(v)\right)^{r_{1}-1} \mathscr{T}^{r_{1} ; \xi}\left(\rho_{2}, v\right) \tilde{\mathcal{g}}(v) d v \\
& +\int_{\rho_{1}}^{\rho_{2}} \xi^{\prime}(v)\left(\xi\left(\rho_{2}\right)-\xi(v)\right)^{r_{1}-1} \mathscr{T}^{r_{1} \xi}\left(\rho_{2}, v\right) \tilde{\mathscr{V}}(v) d v \\
& +\int_{0}^{\rho_{1}} \xi^{\prime}(v)\left(\xi\left(\rho_{1}\right)-\xi(v)\right)^{r_{1}-1} \mathscr{T}^{r_{1} ;}\left(\rho_{2}, v\right) \tilde{\xi}(v) d v \\
& -\int_{0}^{\rho_{1}} \xi^{\prime}(v)\left(\xi\left(\rho_{1}\right)-\xi(v)\right)^{r_{1}-1} \mathscr{T}^{r_{1} ;}\left(\rho_{2}, v\right) \tilde{\mathscr{\delta}}(v) d v \\
& -\int_{0}^{\rho_{1}} \xi^{\prime}(v)\left(\xi\left(\rho_{1}\right)-\xi(v)\right)^{r_{1}-1} \mathscr{T}^{r_{1} ; \xi}\left(\rho_{1}, v\right) \tilde{\mathcal{G}}(v) d v \| \\
& \leq\left\|\delta^{r_{1} ; \xi}\left(\rho_{2}, 0\right) \sigma_{0}-\delta^{r_{1} ; \xi}\left(\rho_{1}, 0\right) \sigma_{0}\right\| \\
& +\left\|\int_{\rho_{1}}^{\rho_{2}} \xi^{\prime}(v)\left(\xi\left(\rho_{2}\right)-\xi(v)\right)^{r_{1}-1} \mathscr{T}^{r_{1} ; \xi}\left(\rho_{2}, v\right) \tilde{\wp}(v) d v\right\| \\
& +\left\|\int_{0}^{\rho_{1}} \xi^{\prime}(v)\left[\left(\xi\left(\rho_{2}\right)-\xi(v)\right)^{r_{1}-1}-\left(\xi\left(\rho_{1}\right)-\xi(v)\right)^{r_{1}-1}\right] \mathscr{T}^{r_{1} \xi \xi}\left(\rho_{2}, v\right) \tilde{\xi}(v) d v\right\| \\
& +\left\|\int_{0}^{\rho_{1}} \xi^{\prime}(v)\left(\xi\left(\rho_{1}\right)-\xi(v)\right)^{r_{1}-1}\left[\mathscr{T}^{r_{1} ; \xi}\left(\rho_{2}, v\right)-\mathscr{T}^{r_{1} ; \xi}\left(\rho_{1}, v\right)\right] \tilde{\mathfrak{g}}(v) d v\right\| \\
& =\mathscr{J}_{1}+\mathscr{I}_{2}+\mathscr{I}_{3}+\mathscr{I}_{4} \text {. }
\end{aligned}
$$

From Lemma 13, it is obvious that $\mathscr{I}_{1} \longrightarrow 0$ as $\rho_{1} \longrightarrow$ $\rho_{2}$ and we get

$$
\begin{aligned}
& \mathscr{I}_{2} \leq \frac{\mathscr{M}\left\|\tilde{\ell}_{1}\right\|_{\infty} \tilde{\ell}_{2}(r)}{\Gamma\left(r_{1}+1\right)}\left(\xi\left(\rho_{2}\right)-\xi\left(\rho_{1}\right)\right)^{r_{1}}, \\
& \mathscr{I}_{3} \leq \frac{\mathscr{M}\left\|\tilde{\ell}_{1}\right\|_{\infty} \tilde{\ell}_{2}(r)}{\Gamma\left(r_{1}+1\right)}\left[\left(\xi\left(\rho_{2}\right)\right)^{r_{1}}-\left(\xi\left(\rho_{1}\right)\right)^{r_{1}}-\left(\xi\left(\rho_{2}\right)-\xi\left(\rho_{1}\right)\right)^{r_{1}}\right]
\end{aligned}
$$

as $\rho_{1} \longrightarrow \rho_{2}$, which yields to $\mathscr{I}_{2} \longrightarrow 0$ and $\mathscr{I}_{3} \longrightarrow 0$. For $\rho_{1}=0$ and $\rho_{2} \in(0, \mathfrak{I}]$, it is clear that $\mathscr{J}_{4}=0$. Then, for any $0<\varepsilon<\rho_{1}$, we get

$$
\begin{aligned}
\mathscr{F}_{4} \leq & \left\|\int_{0}^{\rho_{1}-\varepsilon} \xi^{\prime}(v)\left(\xi\left(\rho_{1}\right)-\xi(v)\right)^{r_{1}-1}\left[\mathscr{T}^{r_{1} ;}\left(\rho_{2}, v\right)-\mathscr{T}^{r_{1} \xi}\left(\rho_{1}, v\right)\right] \tilde{\mathfrak{S}}(v) d v\right\| \\
& +\left\|\int_{\rho_{1}-\varepsilon}^{\rho_{1}} \xi^{\prime}(v)\left(\xi\left(\rho_{1}\right)-\xi(v)\right)^{r_{1}-1}\left[\mathscr{T}^{r_{1} ; \xi}\left(\rho_{2}, v\right)-\mathscr{T}^{r_{1} ; \xi}\left(\rho_{1}, v\right)\right] \tilde{\mathfrak{Q}}(v) d v\right\| \\
\leq & \frac{\left\|\tilde{\ell}_{1}\right\| \tilde{\ell}_{2}(r)}{r_{1}}\left[\left(\xi\left(\rho_{1}\right)-\xi(0)\right)^{r_{1}}-\left(\xi\left(\rho_{1}\right)-\xi\left(\rho_{1}-\varepsilon\right)\right)^{r_{1}}\right] \\
& \times\left(\sup _{0 \leq \varepsilon<\rho_{1}}\left\|\mathscr{T}^{r_{1} ; \xi}\left(\rho_{2}, v\right)-\mathscr{T}^{r_{1} ; \xi}\left(\rho_{1}, v\right)\right\|\right) \\
& +\frac{2 \mathscr{M}\left\|\tilde{\ell}_{1}\right\|_{\infty} \tilde{\ell}_{2}(r)}{\Gamma\left(r_{1}+1\right)}\left[\left(\xi\left(\rho_{1}\right)-\xi\left(\rho_{1}-\varepsilon\right)\right)^{r_{1}}\right],
\end{aligned}
$$

as $\rho_{1} \longrightarrow \rho_{2}$ and $\varepsilon \longrightarrow 0$, which yields to $\mathscr{I}_{4} \longrightarrow 0$; thus,

$$
\left\|\phi\left(\rho_{2}\right)-\phi\left(\rho_{1}\right)\right\| \longrightarrow 0 .
$$

Hence, $\mathfrak{F}\left(B_{r}\right)$ is equicontinuous.

(Step 4) We show that for each $\rho \in \mathfrak{I}^{*}, \mathscr{H}(\rho)=\left\{\left(\mathfrak{F}_{\varepsilon, \delta} \sigma\right.\right.$ ) $\left.(\rho): \sigma \in B_{r}\right\}$ is relatively compact in $\mathscr{X}$.

Clearly, $\mathscr{H}(0)$ is relatively compact in $\mathscr{X}$. Let $\rho \in \mathfrak{J}^{*}$ be fixed, and for any $\varepsilon, \delta>0$ and $\sigma \in B_{r}$, define

$$
\begin{aligned}
& \mathfrak{F}(\sigma)=\int_{0}^{\infty} \vartheta_{r_{1}}(\theta) \mathscr{T}\left((\xi(\rho)-\xi(v))^{r_{1}} \theta\right) \sigma_{0} d \theta \\
& +r_{1} \int_{0}^{\rho-\varepsilon} \int_{\delta}^{\infty} \xi^{\prime}(v)(\xi(\rho)-\xi(v))^{r_{1}-1} \theta \vartheta_{r_{1}}(\theta) \\
& \times \mathscr{T}\left((\xi(\rho)-\xi(v))^{r_{1}} \theta\right) \wp^{\sim}(v) d \theta d v \\
& =\int_{0}^{\infty} \mathcal{\vartheta}_{r_{1}}(\theta) \mathscr{T}\left((\xi(\rho)-\xi(v))^{r_{1}} \theta\right) \sigma_{0} d \theta \\
& +r_{1} \int_{0}^{\rho-\varepsilon} \int_{\delta}^{\infty} \xi^{\prime}(v)(\xi(\rho)-\xi(v))^{r_{1}-1} \theta \vartheta_{r_{1}}(\theta) \\
& \times \mathscr{T}\left((\xi(\rho)-\xi(v))^{r_{1}} \theta+\varepsilon^{r_{1}} \delta-\varepsilon^{r_{1}} \delta\right) \wp^{\sim}(v) d \theta d v \\
& =\int_{0}^{\infty} \vartheta_{r_{1}}(\theta) \mathscr{T}\left((\xi(\rho)-\xi(v))^{r_{1}} \theta\right) \sigma_{0} d \theta \\
& +r_{1} \int_{0}^{\rho-\varepsilon} \int_{\delta}^{\infty} \xi^{\prime}(v)(\xi(\rho)-\xi(v))^{r_{1}-1} \theta \vartheta_{r_{1}}(\theta) \\
& \times\left[\mathscr{T}\left(\mathcal{\varepsilon}^{r_{1}} \delta\right) \mathscr{T}\left((\xi(\rho)-\xi(v))^{r_{1}} \theta-\varepsilon^{r_{1}} \delta\right)\right] \mathcal{\delta}^{\sim}(v) d \theta d v \\
& =\int_{0}^{\infty} \vartheta_{r_{1}}(\theta) \mathscr{T}\left((\xi(\rho)-\xi(v))^{r_{1}} \theta\right) \sigma_{0} d \theta \\
& +r_{1} \mathscr{T}\left(\varepsilon^{r_{1}} \delta\right) \int_{0}^{\rho-\varepsilon} \int_{\delta}^{\infty} \xi^{\prime}(v)(\xi(\rho)-\xi(v))^{r_{1}-1} \theta \vartheta_{r_{1}}(\theta) \\
& \times\left[\mathscr{T}\left((\xi(\rho)-\xi(v))^{r_{1}} \theta-\varepsilon^{r_{1}} \delta\right)\right] \wp^{\sim}(v) d \theta d v .
\end{aligned}
$$


Then, by the compactness of $\mathscr{T}\left(\varepsilon^{r_{1}} \delta\right)$ for $\varepsilon^{r_{1}} \delta>0$, we see that the set $\mathscr{H}_{\varepsilon, \delta}(\rho)=\left\{\left(\widetilde{\mho}_{\varepsilon, \delta} \sigma\right)(\rho): \sigma \in B_{r}\right\}$ is relatively compact in $\mathscr{X}$ for all $\varepsilon, \delta>0$. Moreover, for each $\sigma \in B_{r}$, we have

$$
\begin{aligned}
& \left\|(\mathfrak{\mho} \sigma)(\rho)-\left(\mathfrak{\mho}_{\varepsilon, \delta} \sigma\right)(\rho)\right\|=r_{1} \| \int_{0}^{\rho} \int_{0}^{\delta} \xi^{\prime}(v)(\xi(\rho)-\xi(v))^{r_{1}-1} \theta \vartheta_{r_{1}}(\theta) \mathscr{T}\left((\xi(\rho)-\xi(v))^{r_{1}} \theta\right) \tilde{\mathscr{\wp}}(v) d \theta d v \\
& +\int_{0}^{\rho} \int_{\delta}^{\infty} \xi^{\prime}(v)(\xi(\rho)-\xi(v))^{r_{1}-1} \theta \vartheta_{r_{1}}(\theta) \mathscr{T}\left((\xi(\rho)-\xi(v))^{r_{1}} \theta\right) \tilde{\wp}(v) d \theta d v \\
& -\int_{0}^{\rho-\varepsilon} \int_{\delta}^{\infty} \xi^{\prime}(v)(\xi(\rho)-\xi(v))^{r_{1}-1} \theta \vartheta_{r_{1}}(\theta) \mathscr{T}\left((\xi(\rho)-\xi(v))^{r_{1}} \theta\right) \tilde{\wp}(v) d \theta d v \| \\
& \leq r_{1}\left\|\int_{0}^{\rho} \int_{0}^{\delta} \xi^{\prime}(v)(\xi(\rho)-\xi(v))^{r_{1}-1} \theta \vartheta_{r_{1}}(\theta) \mathscr{T}\left((\xi(\rho)-\xi(v))^{r_{1}} \theta\right) \tilde{\wp}(v) d \theta d v\right\| \\
& +r_{1}\left\|\int_{\rho-\varepsilon}^{\rho} \int_{\delta}^{\infty} \xi^{\prime}(v)(\xi(\rho)-\xi(v))^{r_{1}-1} \theta \vartheta_{r_{1}}(\theta) \mathscr{T}\left((\xi(\rho)-\xi(v))^{r_{1}} \theta\right) \tilde{\mathfrak{\wp}}(v) d \theta d v\right\| \\
& \leq r_{1} \mathscr{M}\left\|\tilde{\ell}_{1}\right\|_{\infty} \tilde{\ell}_{2}(r)\left(\int_{0}^{\rho} \xi^{\prime}(v)(\xi(\rho)-\xi(v))^{r_{1}-1} d v\right)\left(\int_{0}^{\delta} \theta \vartheta_{r_{1}}(\theta) d \theta\right) \\
& +r_{1} \mathscr{M}\left\|\tilde{\ell}_{1}\right\|_{\infty} \tilde{\ell}_{2}(r)\left(\int_{\rho-\varepsilon}^{\rho} \xi^{\prime}(v)(\xi(\rho)-\xi(v))^{r_{1}-1} d v\right)\left(\int_{\delta}^{\infty} \theta \vartheta_{r_{1}}(\theta) d \theta\right) \\
& \leq r_{1} \mathscr{M}\left\|\tilde{\ell}_{1}\right\|_{\infty} \tilde{\ell}_{2}(r)\left(\int_{0}^{\rho} \xi^{\prime}(v)(\xi(\rho)-\xi(v))^{r_{1}-1} d v\right)\left(\int_{0}^{\delta} \theta \vartheta_{r_{1}}(\theta) d \theta\right) \\
& +r_{1} \mathscr{M}\left\|\tilde{\ell}_{1}\right\|_{\infty} \tilde{\ell}_{2}(r)\left(\int_{\rho-\varepsilon}^{\rho} \xi^{\prime}(v)(\xi(\rho)-\xi(v))^{r_{1}-1} d v\right)\left(\int_{0}^{\infty} \theta \vartheta_{r_{1}}(\theta) d \theta\right) \\
& \leq \mathscr{M}\left\|\tilde{\ell}_{1}\right\|_{\infty} \tilde{\ell}_{2}(r)(\xi(\rho)-\xi(0))^{r_{1}}\left(\int_{0}^{\delta} \theta \vartheta_{r_{1}}(\theta) d \theta\right) \\
& +\frac{\mathscr{M}\left\|\tilde{\ell}_{1}\right\|_{\infty} \tilde{\ell}_{2}(r)}{\Gamma(r+1)}(\xi(\rho)-\xi(\rho-\varepsilon))^{r_{1}} \longrightarrow 0, \quad \text { as } \varepsilon, \delta \longrightarrow 0^{+} .
\end{aligned}
$$

Hence, there are relatively compact sets arbitrarily close to $\mathscr{H}(\rho)$ for $\rho>0$, Therefore, $\mathscr{H}(\rho)$ is relatively compact in $\mathscr{X}$.

From the Arzela-Ascoli theorem, $\mathfrak{F}\left(B_{r}\right)$ is relatively compact in $\mathscr{E}$. Consequently, $\mathfrak{F}(\sigma)$ is relatively compact in $\forall \sigma \in \mathscr{E}$.

\section{An Example}

Let $\mathscr{X}=L^{2}([0, \pi])$. Consider the following fractional partial differential inclusions with Caputo derivative $(\xi(\rho)=\rho)$ :

$\left\{\begin{array}{l}{ }^{C} D_{a+}^{1 / 2 ; \rho} \sigma(\rho, v) \in \frac{\partial^{2}}{\partial v^{2}} \sigma(\rho, v)+f(\rho, \sigma(\rho, v)), \quad \rho \in(0,1), \sigma \in(0, \pi], \\ \sigma(\rho, 0)=\sigma(\rho, \pi)=0, \quad \sigma(0, v)=\sigma_{0},\end{array}\right.$

where $r_{1}=1 / 2$ and $\mathscr{T}=1$, and we define an operator $\mathscr{A}$ by $\mathscr{A} \sigma=\sigma^{\prime \prime}$ for $\sigma \in \mathfrak{D}(\mathscr{A})$, where $\mathfrak{D}(\mathscr{A})=\left\{\sigma \in \mathscr{X}: \sigma, \sigma^{\prime}\right.$ are absolutely continuous, and $\sigma(0)=\sigma(\pi)=0\}$. Then, $\mathscr{A}$ generates a strongly continuous semigroup $\{\mathscr{T}(\rho)\}_{\rho \geq 0}$ which is compact. Furthermore, $\|\mathscr{T}(\rho)\| \leq e^{-\rho} \leq 1=\mathscr{M}, \quad \rho \geq 0$. Let $f:[0,1] \times \mathbb{R} \longrightarrow \mathcal{O}(\mathbb{R})$ defined by

$$
\sigma(\rho, v) \longrightarrow f(\rho, \sigma(\rho, v))=\left[0, \frac{\sin (\sigma(\rho, v))}{\left(\exp \left(\rho^{2}\right)+2\right)}+\frac{1}{15}\right]
$$

Clearly, $H_{d}(f(\rho, \sigma), f(\rho, \bar{\sigma})) \leq \mathfrak{z}(\rho)\|\sigma-\bar{\sigma}\|$, where $\mathfrak{z}(\rho)$ $=1 /\left(\exp \left(\rho^{2}\right)+2\right)$ and $d(0, f(\rho, 0))=1 / 15 \leq \mathfrak{z}(\rho)$ for (a.e.) all $\rho \in[0,1]$. Besides, we obtain $\|\mathfrak{z}\|_{\infty}=1 / 3$ which implies $\rho\left\|_{\mathfrak{z}}\right\| \simeq 0.38<1$. Consequently, all items of Theorem 16 are satisfied. Then, there exists at least one solution of (10) on $[0,1]$.

Remark 18. Our current results for problem (3) remain true for the following cases:

(i) Caputo-type inclusion problem: $\xi(\rho)=\rho$.

(ii) Caputo-Hadamard-type inclusion problem: $\xi(\rho)=$ $\log (\rho)$.

(iii) Generalized Caputo-type inclusion problem: $\xi(\rho)$ $=\rho^{\rho}, \rho>0$. 
Remark 19. The obtained results for problem (3) include the results of Suechoei and Ngiamsunthorn [17]; i.e., problem (3) is reduced to the considered problem in [17] when $\{f(\rho, \sigma(\rho))\}=h(\rho, \sigma(\rho))$.

\section{Concluding Remarks}

We have studied the class of IVPs for generalized Caputo FDIs with Lipschitz set-valued functions. We obtained the existence result by using the FPT of Covitz and Nadler. Also, we characterized the topological properties of the set of solutions. We confirm that our acquired outcomes are new in the frame of generalized Caputo FDIs with initial conditions and it greatly contributes to enriching the existing literature on this theme.

In future works, many cases can be established when one takes a more generalized operator, for example, $\psi$-Hilfer fractional operator [13]. Further, it will be of interest to study the existing problem in this article for the MittagLeffler power law [8] and for fractal fractional operators [35].

\section{Data Availability}

No data were used to support this study.

\section{Conflicts of Interest}

The authors declare that they have no competing interests.

\section{Authors' Contributions}

All authors made equal contributions and read and supported the last original copy.

\section{Acknowledgments}

The authors are grateful for the support provided by their institutions.

\section{References}

[1] Y. Benia and A. Scapellato, "Existence of solution to Kortewegde Vries equation in a non-parabolic domain," Nonlinear Analysis, vol. 195, article 111758, 2020.

[2] L. Debnath, "Recent applications of fractional calculus to science and engineering," International Journal of Mathematics and Mathematical Sciences, vol. 2003, no. 54, pp. 3413-3442, 2003.

[3] A. Scapellato, "Riesz potential, Marcinkiewicz integral and their commutators on mixed Morrey spaces," Filomat, vol. 34, no. 3, pp. 931-944, 2020.

[4] J. Singh, "Analysis of fractional blood alcohol model with composite fractional derivative," Chaos Solitons \& Fractals, vol. 140, article 110127, 2020.

[5] N. D. Phuong, L. V. C. Hoan, E. Karapinar, J. Singh, H. D. Binh, and N. H. Can, "Fractional order continuity of a time semi-linear fractional diffusion-wave system," Alexandria Engineering Journal, vol. 59, no. 6, pp. 4959-4968, 2020.

[6] J. Singh, B. Ganbari, D. Kumar, and D. Baleanu, "Analysis of fractional model of guava for biological pest control with memory effect," Journal of Advanced Research, vol. 32, pp. 99-108, 2021.

[7] R. Almeida, "A Caputo fractional derivative of a function with respect to another function," Communications in Nonlinear Science and Numerical Simulation, vol. 44, pp. 460-481, 2017.

[8] A. Atangana and D. Baleanu, "New fractional derivatives with nonlocal and non-singular kernel: theory and application to heat transfer model," Thermal Science, vol. 20, no. 2, pp. 763-769, 2016.

[9] M. Caputo and M. Fabrizio, "A new definition of fractional derivative without singular kernel," Progress in Fractional Differentiation and Applications, vol. 1, no. 2, pp. 73-85, 2015.

[10] F. Jarad, T. Abdeljawad, and D. Baleanu, "Caputo-type modification of the Hadamard fractional derivatives," Advances in Difference Equations, vol. 142, 2012.

[11] F. Jarad and T. Abdeljawad, "Generalized fractional derivatives and Laplace transform," Discrete \& Continuous Dynamical Systems-S, vol. 13, no. 3, pp. 709-722, 2020.

[12] U. N. Katugampola, "A new approach to generalized fractional derivatives," Bulletin of Mathematical Analysis \& Applications, vol. 6, no. 4, pp. 1-15, 2014.

[13] J. Vanterler da C Sousa and E. Capelas de Oliveira, "On the $\psi$ Hilfer fractional derivative," Communications in Nonlinear Science and Numerical Simulation, vol. 60, pp. 72-91, 2018.

[14] P. Bedi, A. Kumar, T. Abdeljawad, and A. Khan, "Existence of mild solutions for impulsive neutral Hilfer fractional evolution equations," Advances in Difference Equations, vol. 2020, no. 1, 2020.

[15] P. Bedi, A. Kumar, T. Abdeljawad, and A. Khan, "Study of Hilfer fractional evolution equations by the properties of controllability and stability," Alexandria Engineering Journal, vol. 60, no. 4, pp. 3741-3749, 2021.

[16] P. Chen, Y. Li, Q. Chen, and B. Feng, "On the initial value problem of fractional evolution equations with noncompact semigroup," Computers \& Mathematics with Applications, vol. 67, no. 5, pp. 1108-1115, 2014.

[17] A. Suechoei and P. S. Ngiamsunthorn, "Existence uniqueness and stability of mild solutions for semilinear $\psi$-Caputo fractional evolution equations," Advances in Difference Equations, vol. 2020, no. 1, pp. 1-28, 2020.

[18] Y. Zhou and F. Jiao, "Existence of mild solutions for fractional neutral evolution equations," Computers \& Mathematics with Applications, vol. 59, no. 3, pp. 1063-1077, 2010.

[19] M. S. Abdo, S. T. Thabet, and B. Ahmad, "The existence and Ulam-Hyers stability results for $\psi$-Hilfer fractional integrodifferential equations," Journal of Pseudo-Differential Operators and Applications, vol. 11, no. 4, pp. 1757-1780, 2020.

[20] M. A. Alqudah, C. Ravichandran, T. Abdeljawad, and N. Valliammal, "New results on Caputo fractional-order neutral differential inclusions without compactness," Advances in Difference Equations, vol. 2019, no. 1, pp. 1-14, 2019.

[21] S. Belmor, F. Jarad, T. Abdeljawad, and M. A. Alqudah, "On fractional differential inclusion problems involving fractional order derivative with respect to another function," Fractals, vol. 28, no. 8, article 2040002, 2020.

[22] A. Lachouri, A. Ardjouni, and A. Djoudi, "Investigation of the existence and uniqueness of solutions for higher order fractional differential inclusions and equations with integral boundary conditions," Journal of Interdisciplinary Mathematics, vol. 2021, pp. 1-19, 2021. 
[23] A. Lachouri, M. S. Abdo, A. Ardjouni, B. Abdalla, and T. Abdeljawad, "Hilfer fractional differential inclusions with Erdélyi-Kober fractional integral boundary condition," Advances in Difference Equations, vol. 2021, no. 1, pp. 1-17, 2021.

[24] A. A. Kilbas, H. M. Srivastava, and J. J. Trujillo, Theory and Applications of Fractional Differential Equations, vol. 204 of North-Holland Mathematics Studies, Elsevier, Amsterdam, Netherlands, 2006.

[25] S. G. Samko, A. A. Kilbas, and O. I. Marichev, Fractional Integrals and Derivatives: Theory and Applications, Gordon \& Breach, Yverdon, 1993.

[26] F. Mainardi, "On the initial value problem for the fractional diffusion-wave equation," in Waves and Stability in Continuous Media, vol. 1994, pp. 246-251, World Scientific, Singapore, 1994.

[27] I. Podlubny, Fractional Differential Equations, Academic Press, San Diego, 1999.

[28] K. Engel and R. Nagel, One-Parameter Semigroups for Linear Evolution Equations, Springer, New York, 2000.

[29] A. Pazy, Semigroups of Linear Operators and Applications to Partial Differential Equations, Springer, New York, 1983.

[30] K. Deimling, "Set-valued differential equations," De Gruyter, Berlin, 1992.

[31] S. Hu and N. Papageorgiou, Handbook of Multivalued Analysis, Vol I: Theory, Kluwer, Dordrecht, 1997.

[32] H. Covitz and S. B. Nadler Jr., "Multivalued contraction mappings in generalized metric spaces," Israel Journal of Mathematics, vol. 8, no. 1, pp. 5-11, 1970.

[33] M. Kisielewicz, Differential Inclusions and Optimal Control, Kluwer, Dordrecht, The Netherlands, 1991.

[34] C. Castaing and M. Valadier, Convex Analysis and Measurable Multifunctions, Springer, Berlin/Heidelberg, Germany; New York, NY, USA, 1977.

[35] A. Atangana, "Fractal-fractional differentiation and integration: connecting fractal calculus and fractional calculus to predict complex system," Chaos Solitons \& Fractals, vol. 102, pp. 396-406, 2017. 